\title{
Motivational influences on compliance with and consequences of instructions to suppress stereotypes
}

\section{Wyer, NA}

http://hdl.handle.net/10026.1/2953

\subsection{6/j.jesp.2006.03.001 \\ Journal of Experimental Social Psychology}

All content in PEARL is protected by copyright law. Author manuscripts are made available in accordance with publisher policies. Please cite only the published version using the details provided on the item record or document. In the absence of an open licence (e.g. Creative Commons), permissions for further reuse of content should be sought from the publisher or author. 


\title{
Motivational influences on compliance with and consequences of instructions to suppress stereotypes
}

\author{
Natalie A. Wyer \\ School of Psychology, University of Plymouth, Drake Circus, Plymouth PL4 8AA, UK \\ Received 21 November 2005; revised 5 March 2006 \\ Available online 2 May 2006
}

\begin{abstract}
Two experiments investigated differences in compliance with instructions to suppress stereotypes as a function of prejudice-related motivations. In Experiment 1, only participants identified as high in motivation to control prejudice [Dunton, B. C., \& Fazio, R. H. (1997). An individual difference measure of motivation to control prejudiced reactions. Personality and Social Psychology Bulletin, 23, 316-326] complied with suppression instructions. These participants experienced post-suppression rebound effects, but only if they were also high in prejudice. In Experiment 2, only participants identified as high in external motivation to respond without prejudice [Plant, E. A., \& Devine, P. G. (1998). Internal and external motivation to respond without prejudice. Journal of Personality and Social Psychology, 75, 811-832] complied with instructions to suppress. These participants later experienced stereotype rebound effects, but only if they were also low in internal motivation to respond without prejudice. These findings suggest that motivational factors play an important role in determining not only the outcome of suppression, but also the choice to attempt suppression in the first place.
\end{abstract}

(c) 2006 Elsevier Inc. All rights reserved.

Keywords: Stereotype suppression; Prejudice; Motivation

\section{Introduction}

For a variety of reasons, individuals may wish to avoid acting upon cultural stereotypes. Recent research has identified a number of processes that may allow people to accomplish this goal. For example, taking the perspective of outgroup members may decrease one's use of related stereotypes (Galinksy \& Moskowitz, 2000). Further, people with egalitarian goals may inhibit stereotypes at a pre-conscious level (Moskowitz, Gollwitzer, Wasel, \& Schaal, 1999).

Perhaps the most well-researched way to avoid stereotypic influences is by engaging in stereotype suppression (i.e., actively avoiding stereotypic thoughts). Research has demonstrated that this strategy is spontaneously adopted in a variety of circumstances (Macrae, Bodenhausen, \& Milne, 1998; Wyer, Sherman, \& Stroessner, 1998). Ironically, how-

E-mail address: natalie.wyer@plymouth.ac.uk. ever, the very attempt to suppress stereotypic thoughts is often followed by an increase ('rebound') in the accessibility of those thoughts (Macrae, Bodenhausen, Milne, \& Jetten, 1994; Wyer, Sherman, \& Stroessner, 2000).

Following the initial demonstration of stereotype rebound effects (Macrae et al., 1994), researchers began seeking potential moderators of the effect. Many of these endeavors have focused on the possibility that individuals with unprejudiced beliefs or sufficient motivation to behave in unprejudiced ways may succeed in circumventing stereotype rebound effects (Gordijn, Hindriks, Koomen, Dijksterhuis, \& van Knippenberg, 2004; Monteith, Spicer, \& Tooman, 1998b). For example, Monteith et al. (1998b) found rebound effects following the suppression of homosexual stereotypes, but only among participants who were prejudiced against homosexuals (see also Hodson \& Dovidio, 2001). Similarly, Gordijn et al. (2004) demonstrated that participants with high levels of internal motivation to respond without prejudice required 
no additional cognitive resources to suppress the stereotype of skinheads, nor did they experience a post-suppression increase in the stereotype's accessibility.

\section{Individual differences in prejudice-related motivations}

The research described above suggests that individual differences in beliefs and motivations may moderate the outcome of suppression attempts when they occur. However, these factors may also influence whether or not suppression is attempted in the first place. At least two constructs relating to prejudice-related motivation have been developed in recent years. First, Dunton and Fazio (1997) introduced the 'motivation to control prejudice' construct, which incorporates concern with acting prejudiced and restraint to avoid dispute. Similarly, Plant and Devine (1998) introduced a measure of internal and external motivation to respond without prejudice.

Both Dunton and Fazio's (1997) motivation to control prejudice and Plant and Devine's (1998) motivation to respond without prejudice have been associated with a variety of important responses. Relative to those low in motivation to control prejudice, high motivation individuals respond in more discrepant ways to overt versus unobtrusive measures of racial attitudes (Fazio, Jackson, Dunton, \& Williams, 1995), experience greater emotional distress after behaving in a prejudiced manner (Fazio \& Hilden, 2001), are less likely to have had positive past experiences with outgroup members, and are less comfortable with unscripted interactions with outgroup members (Towles-Schwen \& Fazio, 2003). In research stemming from Plant and Devine's (1998) distinction between internal and external motivation to respond without prejudice, participants who are primarily motivated by internal sources report more positive racial attitudes on explicit measures (Devine, Plant, Amodio, Harmon-Jones, \& Vance, 2002) and have more positive expectations concerning future interactions with outgroup members (Plant, 2004). Conversely, participants who are primarily motivated by external sources are more likely to reveal discrepant racial attitudes on implicit versus explicit measures (Devine et al., 2002) and experience reactance when confronted with external pressures to behave in unprejudiced ways (Plant \& Devine, 2001).

\section{Motivational factors and the adoption of suppression goals}

The present research investigates the possibility that individual differences in motivation predict compliance with external suppression demands. In two experiments, motivation to control prejudice (Experiment 1) and motivation to respond without prejudice (Experiment 2) were considered as moderators of not only the outcome of suppression attempts, but also compliance with instructions to suppress. With few exceptions (e.g., Macrae et al., 1998; Wyer et al., 1998), influences on the adoption of suppression goals have not been investigated. Moreover, the likeli- hood that some individuals resist external pressures to suppress (e.g., instructions to avoid stereotyping) has not been examined. ${ }^{1}$

The present experiments test the hypothesis that individual differences in motivation to behave in non-prejudiced ways predict the extent to which people comply with external demands to suppress their stereotypes. Thus, it is expected that only participants possessing sufficient motivation will suppress their stereotypes when instructed to do so. Among those participants who do comply with suppression instructions, individual differences in internal sources of prejudicerelated motivations are expected to moderate the extent to which post-suppression rebound effects occur.

\section{Experiment 1}

When confronted with situational cues to avoid stereotyping, individuals' responses may depend on their motivation to control prejudiced responses (Dunton \& Fazio, 1997). Thus, whereas highly motivated individuals may conform to situational suppression cues, unmotivated individuals may ignore or oppose the same cues. Consequently, in the context of experimental instructions to suppress stereotypes, highly motivated participants may be expected to comply with those instructions, while those low in motivation may decline to do so.

If compliance with suppression instructions varies as a function of motivation to control prejudice, what are the implications for stereotype rebound? Research by Monteith et al. (1998b) suggests that participants' level of prejudice moderates their experience of rebound effects. In their research, post-suppression rebound occurred only among participants with highly prejudiced beliefs. As suggested by Monteith and her colleagues, stereotypes may be automatically activated among highly prejudiced individuals, leading them to recruit a wide variety of distracters to replace stereotypic thoughts during suppression. These 'replacement thoughts' may subsequently trigger the retrieval of stereotypic thoughts, thereby resulting in stereotype rebound. In contrast, stereotypes may not be automatically activated for unprejudiced individuals. Furthermore, when unprejudiced individuals do suppress stereotypic thoughts, they are better able to produce replacement thoughts (e.g., egalitarian beliefs) which are unlikely to trigger retrieval of the suppressed stereotype later.

\footnotetext{
${ }^{1}$ Previous research (e.g., Gordijn et al., 2004; Monteith et al., 1998b) is inconsistent with regard to individual differences in compliance with suppression instructions. Monteith et al. (1998b) found that unprejudiced participants generated few stereotypic thoughts regardless of suppression instructions. In contrast, Gordijn and colleagues reported that internal motivation did not moderate the extent to which participants suppressed skinhead stereotypes when instructed to do so. Gordijn et al., do not report analyses involving external motivation, which might be expected to be a more important predictor of compliance rates. Indeed, external pressures to avoid using the stereotype of skinheads are likely minimal; thus, the researchers may have found little variance in participants' level of external motivation.
} 
Thus, past research suggests that the interaction between prejudice and the motivation to control it should determine the outcome of instructions to engage in stereotype suppression. In the present experiment, both prejudice and motivation to control prejudice were measured prior to a standard stereotype suppression experiment. Participants engaged in an impression formation task involving a Black target, while suppressing their stereotypes or not. Participants high in motivation to control prejudice were expected to comply with instructions to suppress, whereas those low in motivation were expected to resist doing so. In a second phase of the experiment, participants were asked to form an impression of another individual based on an ambiguously hostile description. Participants who had previously suppressed their stereotypes (i.e., highly motivated participants in the suppression condition) should be influenced by accessible stereotypes of Blacks and judge this target as more hostile. That is, they should experience stereotype rebound. However, this effect was expected to occur only among participants who were also high in prejudice against Blacks.

\section{Method}

\section{Participants}

Ninety-four undergraduate students in an introductory psychology course at the University of California, Santa Barbara participated in the experiment in exchange for partial credit towards a course requirement. Participants were recruited from a pool of students who took part in an earlier mass testing session in which they completed the Motivation to Control Prejudiced Responses scale (MCPR, Dunton \& Fazio, 1997) and the Modern Racism Scale (MRS, McConahay, 1986).

\section{Design and procedure}

The experiment consisted of two phases: a suppression phase and an expression phase. To create the illusion that the two phases were unrelated, each was conducted in a different laboratory and by a different experimenter. In the suppression phase, a female experimenter informed participants that they would complete a number of unrelated studies during the session. The first study was described as investigating how people form impressions of others based on visual information. Participants were shown a photograph of a Black male (approximately age 25), and were asked to write a description of a typical day in his life. Participants in the suppression condition were cautioned that, because research had shown that people were often influenced by stereotypes when forming impressions, they should avoid thinking about stereotypes while they wrote. Participants in the control condition received no such instructions. Participants were allowed $5 \mathrm{~min}$ to write, after which they were thanked for their participation and directed to another room where the expression phase of the experiment was conducted.

In the expression phase, a female experimenter instructed participants that the study concerned how peo- ple form impressions of others based on written information. Participants were informed that they would read a short description of someone and that they should form an impression of this person. Participants then read a story about Donald, ${ }^{2}$ whose behavior could be construed as hostile. This story was adapted from Srull and Wyer (1979) and is sufficiently ambiguous to allow differing interpretations (e.g., Bargh \& Pietromonaco, 1982; Devine, 1989; Wyer et al., 1998). After reading about Donald, participants rated him on a number of personality traits, including hostilityrelated (hostile, dislikeable, unfriendly, thoughtful, kind, and considerate) and hostility-unrelated (boring, narrowminded, conceited, intelligent, dependable, and interesting) traits.

After completing the expression phase of the experiment, participants were debriefed and thanked for their participation.

\section{Results}

\section{Individual difference measures}

The theoretical range of scores on the MCPR (Dunton $\&$ Fazio, 1997) is from -3 to +3 (with lower numbers indicating lower motivation). The actual range obtained from participants in this experiment was -1.71 to +2.29 $(M=0.28, s=0.87){ }^{3}$ The theoretical range of scores on the MRS (McConahay, 1986) is from -2 to +2 (with lower numbers indicating less prejudice). The actual range among participants in this experiment was -2.0 to +1.0 $(M=-1.00, s=0.79)$. The MRS and MCPR were uncorrelated, $r=-.01$.

\section{Suppression phase}

Two independent coders were given a description of the cultural stereotype of Blacks, which included positive and negative stereotypic attributes. Each coder read each story and rated its stereotypicality on a 1-10 scale. Reliability between the two coders was satisfactory, $\alpha=.76$; thus, their ratings were averaged into a single stereotypicality score.

Regression analyses were conducted on the stereotypicality scores, entering suppression condition (coded as -1 for suppression, +1 for control), MRS scores, and MCPR scores (along with interactions among the three) as predictors. Although there were no main effects of the individual difference measures on stereotype use, there was a significant interaction between the two, $\beta=-.907, p=.01$. Analysis of the simple slopes (calculated at \pm 1 standard deviation from the mean MCPR score) indicated that, among partici-

\footnotetext{
${ }^{2}$ Following previous research employing the Donald story to assess stereotype activation (e.g., Devine, 1989; Wyer et al., 1998, 2000), Donald's race was not identified.

${ }^{3}$ In other research involving motivation to control prejudiced responses, Fazio and colleagues have found different relationships involving the two components of the MCPR scale. Analyses involving the two sub-scales were also examined in this research. However, the two sub-scales had similar effects and interacted similarity with the other variables, and thus will not be discussed separately.
} 


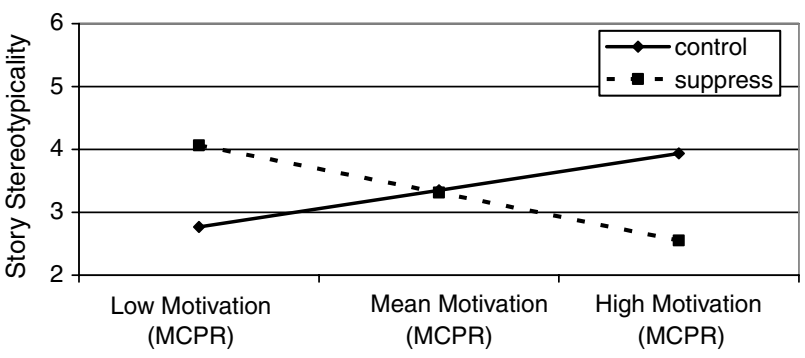

Fig. 1. Experiment 1, suppression phase: mean stereotypicality of stories as a function of suppression condition and participants' scores on the motivation to control prejudiced responses (MCPR) scale. Regression lines estimated at points $-1,0$, and +1 standard deviation from the mean MCPR score.

pants with low MCPR scores, MRS scores significantly predicted stereotypicality scores, $\beta=1.14, p<.01$. In contrast, among participants with higher MCPR scores, MRS did not predict stereotypicality scores $(\beta=-.44, \mathrm{~ns})$.

Regression analyses further revealed that the main effect of suppression condition was not significant ( $\beta=-.03$, ns), indicating that - in general-participants in the suppression condition $(M=3.79, s=2.11)$ did not rely on stereotypes to a lesser extent than those in the control condition $(M=3.85, s=1.85)$. However, there was a significant interaction between MCPR score and suppression condition, $\beta=-.76, p<.01$ (see Fig. 1). Analysis of the simple slopes (computed at \pm 1 standard deviation from the mean MCPR score) indicated that, among lower MCPR participants, suppression instructions generated more stereotypic responses than control instructions, $\beta=.63, p=.05$. In contrast, at higher levels of MCPR, suppression instructions produced significantly less stereotypic responses, $\beta=-.68, p=.02$. No other effects were significant. These results suggest that instructions to suppress were effective only among participants who were motivated to control prejudiced responses. In contrast, unmotivated participants appeared to increase their use of stereotypes in the very conditions where they were instructed to avoid doing so.

\section{Expression phase}

Ratings of Donald on the six hostility-related traits were reliable $(\alpha=.74)$ and thus were averaged to form a single stereotypicality score. Regression analyses were conducted on the stereotypicality scores, entering suppression condition (coded as -1 for suppression, +1 for control), MRS scores, and MCPR scores as predictor variables. The main effect of condition was not significant, $\beta=.02$, ns, indicating that participants in the suppression condition $(M=7.81$, $s=1.24)$ did not differ from those in the control condition $(M=7.82, s=1.12)$. The analyses revealed no other significant main effects, and only one marginally significant twoway interaction between MRS and suppression condition $(\beta=.30, p=.07)$. This interaction was, however, significantly qualified by a three-way interaction involving MCPR, $\beta=.53, p=.01$ (see Fig. 2).
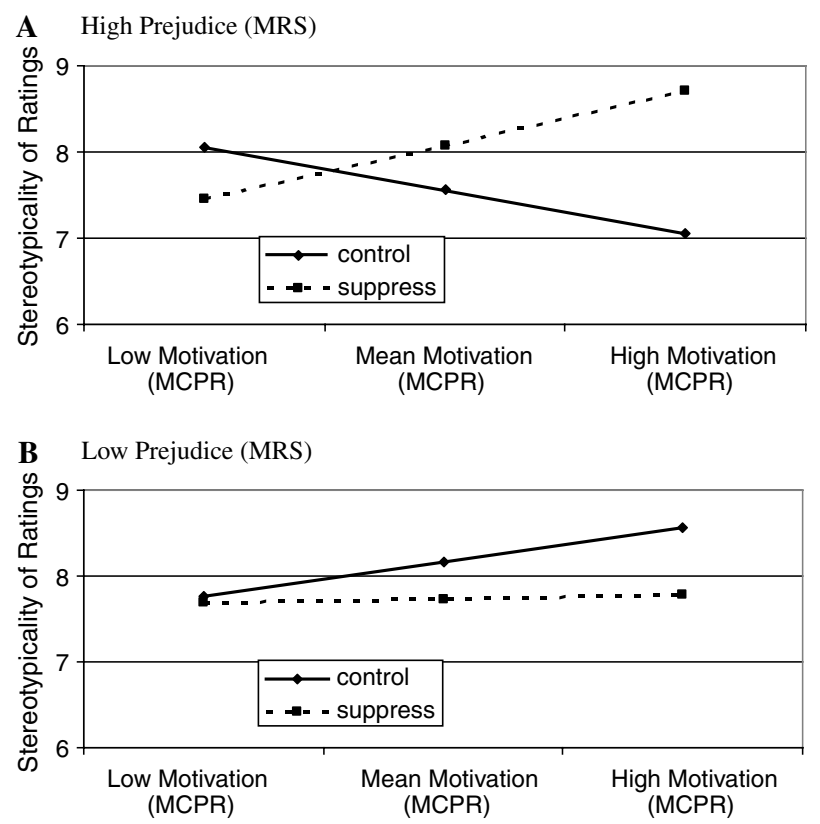

Fig. 2. Experiment 1, expression phase: mean stereotypicality of ratings of Donald as a function of suppression condition and participants' scores on the motivation to control prejudiced responses (MCPR) scale for participants with high scores (+1 standard deviation) on the modern racism scale (MRS; A) and low scores ( -1 standard deviation) on the MRS (B). Regression lines estimated at points $-1,0$, and +1 standard deviation from the mean MCPR score.

Analysis of simple slopes (calculated at \pm 1 standard deviation from the mean MRS and MCPR scores) indicated that suppression condition had no significant effect on stereotype use by participants with low MCPR scores, regardless of their scores on the MRS ( $\beta \mathrm{s}=-.05$ and -.29 , ns, for low and high MRS scorers, respectively). Among participants with higher scores on the MCPR scale, the effect of suppression depended on their MRS scores. For those with lower MRS scores, suppression condition did not significantly predict stereotype use $(\beta=-.39$, ns). In contrast, those with higher MRS evidenced higher stereotype use in the suppression condition than in the control condition, $\beta=.81, p<.01$. Thus, stereotype suppression led to rebound effects for high motivation participants, but only if they were also high in prejudice. No other effects approached significance.

It is interesting to note that participants in the control condition who were both highly prejudiced and highly motivated provided the least stereotypic ratings of Donald, providing a low baseline against which to assess stereotype rebound. However, interpreting differences in stereotype rebound among participants at different ends of these two individual difference dimensions is problematic, as rebound effects are necessarily defined in relative terms. To the extent that a group of participants demonstrates greater stereotype use following suppression than does a group of otherwise similar participants who have not suppressed, a rebound effect is considered to have occurred. There are a number of reasons to suspect that individual differences in stereotype-relevant beliefs and motivations may be associ- 
ated with differences in sensitivity to stereotype-relevant information and the tendency to draw inferences from it (see Biernat \& Manis, 1994 for one example). Thus, the critical comparisons on which to focus are those within the same 'type' of participant.

\section{Discussion}

Motivation to control prejudice appears to play two important roles in the stereotype suppression process. In this experiment, participants who were highly motivated to control prejudice were compliant with experimental demands to suppress their stereotypes. When they were given an explicit goal to avoid stereotyping, they were successful at reducing stereotypic influences on their responses. Their vigilance during the suppression phase, however, appeared to backfire in the expression phase. The very participants who were initially most successful at avoiding stereotype use were later the most likely to be influenced by those stereotypes. Importantly, this effect only emerged among participants who also held relatively prejudiced attitudes. Less prejudiced (but equally motivated) participants, while complying with suppression instructions, were apparently able to avoid post-suppression rebound effects.

In contrast, participants who were relatively unmotivated to control prejudice failed to comply with instructions to suppress their stereotypes and appeared to escalate their use of stereotypes. Consequently, these participants were less influenced by stereotypes during the subsequent impression formation task. Thus, while motivation to control prejudice may contribute to less stereotypic judgments in the short term, highly motivated individuals may fall prey to counter-intentional increases in stereotype use once explicit suppression demands are lifted.

\section{Experiment 2}

Experiment 1 demonstrated that motivational factors influence the extent to which individuals comply with external demands to avoid stereotyping. Whatever its source, motivation to control prejudice led participants to conform to situational suppression cues while those lacking motivation failed to do so. While the measure used in Experiment 1 did not distinguish between internal and external sources of motivation, it is likely that this distinction is important when it comes to one's reactions to external suppression cues. In particular, suppression attempts elicited by an external source (the experimenter) may have different consequences than those instigated by personal choice. One who is motivated by a personal desire to avoid influence by prejudiced beliefs may view external demands to suppress stereotypic thoughts as redundant with their personal motives, and thus be relatively uninfluenced by them.

In contrast, individuals who are motivated primarily by external pressures to avoid responding in a prejudiced manner may be particularly sensitive to an experimenter's suggestion that they should avoid stereotypic thoughts. These individuals, then, may be particularly vulnerable to stereotype rebound effects. Consistent with this idea, Plant and Devine (2001) found that participants generally complied with an initial request to make judgments in favor of a Black target, regardless of their motivations to respond without prejudice (although there was a non-significant tendency for those high in external motivation to be more compliant than those low in external motivation). However, when they were later given the opportunity to make a second (unconstrained) judgment about the same target, participants with the combination of low internal motivation and high external motivation made particularly negative judgments.

In Experiment 2, participants' internal and external motivations to respond without prejudice (Plant \& Devine, 1998) were measured prior to a standard stereotype suppression experiment. Participants were asked to form an impression of a Black target while either suppressing their stereotypes or not. Participants high in external motivation were expected to comply with suppression instructions while those low in external motivation were expected not resist doing so. Participants then completed a word-stem completion task involving a number of stereotypic items. Participants who suppressed their stereotypes (highly externally motivated participants in the suppression condition) were expected to show a rebound in stereotype accessibility, as evidenced by a greater number of Black-stereotypic word stem completions. Following prior research (Gordijn et al., 2004), this effect was only expected to occur among participants who were also low in internal motivation to respond without prejudice.

\section{Method}

\section{Participants}

Participants were 114 undergraduate students at the University of Plymouth, 30 of whom completed the experiment in exchange for course credit. The remaining participants completed the experiment in exchange for £3. Paid participants were evenly distributed across conditions.

\section{Design and procedures}

The experiment consisted of three phases, all conducted by a female experimenter. Participants were informed that they would take part in a number of unrelated studies during the session. In the first phase, participants completed the Internal Motivation (IMS) and External Motivation (EMS) scales (Plant \& Devine, 1998). Participants then completed an unrelated experiment lasting approximately $20 \mathrm{~min}$, after which they completed the suppression and expression phases of this experiment.

In the suppression phase, participants were introduced to a study investigating how people form impressions of others. Participants were shown a photograph of a Black male (approximately age 40), and were asked to write a description of a typical day in his life. Participants in the suppression condition were further instructed that they 
should avoid thinking about or using racial stereotypes while they wrote the description. Participants in the control condition received no such instructions. All participants were allowed $5 \mathrm{~min}$ to write, after which they were informed that the study was finished and that they proceed to the next experiment.

In the expression phase, participants were introduced to a 'language use' task. They were provided with a series of 20 word stems with instructions to complete the stems with the first word that came to mind. The word stems were based on stimuli used by Wittenbrink, Judd, and Park (1997). Ten of the stems could be completed with words associated with the Black stereotype (athletic, playful, charming, musical, fashionable, poor, violent, dishonest, dangerous, lazy). The remaining ten word stems could be completed with words associated with the White stereotype (successful, intelligent, educated, wealthy, responsible, boring, selfish, materialistic, greedy, sheltered).

After completing the expression phase of the experiment, participants were debriefed and thanked for their participation.

\section{Results}

\section{Individual difference measures}

The theoretical range of scores on both the EMS and IMS (Plant \& Devine, 1998) is 1 to 9 (with higher numbers indicating higher motivation). The actual range of EMS scores among participants in this experiment was 2.4 to 7.8 $(M=4.17, s=1.40)$. The actual range of IMS scores was 3.2 to $9.0(M=7.08, s=1.65)$. The EMS and IMS were uncorrelated, $r=-.04$, ns.

\section{Suppression phase}

Two independent coders were given descriptions of the cultural stereotype of Blacks which included both positive and negative stereotypic attributes. The descriptions generated during the suppression phase were rated for stereotypicality by each coder. Ratings were made on a -5 to +5 scale, with negative numbers indicating counter-stereotypic content and positive numbers indicating stereotypic content. The two coders' ratings were reliable $(\alpha=.79)$ and were therefore averaged to form a single stereotypicality score.

Regression analyses were conducted on story stereotypicality scores, entering suppression condition (coded as +1 for suppression, -1 for control), EMS scores, and IMS scores (and their interactions) as predictor variables. As in Experiment 1, there was not a significant main effect of suppression condition, $\beta=-.16$, ns, indicating that, in general, participants did not produce less stereotypic stories under suppression instructions $(M=.06, s=2.16)$ than under control instructions $(M=.47, s=1.60)$. There was, however, a significant main effect of EMS scores, $\beta=-.33, p=.01$, indicating that participants higher in external motivation wrote less stereotypic descriptions than those lower in external motivation. This effect was qualified by a signifi-

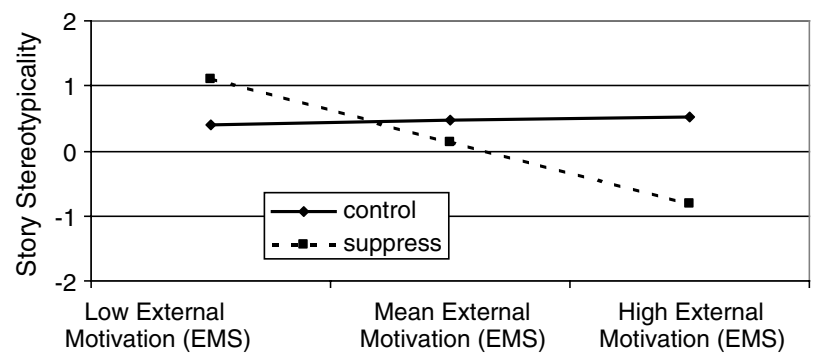

Fig. 3. Experiment 2, suppression phase: mean stereotypicality of stories as a function of suppression condition and participants' scores on the external motivation scale (EMS). Regression lines estimated at points -1 , 0 , and +1 standard deviation from the mean EMS scores.

cant interaction between EMS scores and suppression condition, $\beta=-.36, p<.01$ (see Fig. 3). Analysis of simple slopes (computed at \pm standard deviation from the mean EMS score) indicated that, among participants with lower EMS scores, suppression instructions had no significant impact on stereotypicality scores, $\beta=.34$, ns. In contrast, participants with higher EMS scores wrote significantly less stereotypic stories in the suppression condition than in the control condition, $\beta=-.66, p<.01$. Thus, it appears that participants high in external motivation to respond without prejudice complied with instructions to suppress, whereas those low in external motivation did not. No other main effects or interactions were significant.

\section{Expression phase}

The number of word stems completed with the target word for each category (White positive, White negative, Black positive, and Black negative) was computed. Stem completion rates did not differ as a function of word valence (largest $F(1,106)=1.44, p>.20$ ), thus positive and negative words were summed within each stereotype category.

Regression analyses were conducted on the number of Black-stereotypic stem completions, entering suppression condition (coded as +1 for suppression, -1 for control), EMS scores, IMS scores, and their interactions as predictors. White-stereotypic stem completions were also entered, serving as a covariate. ${ }^{4}$ The analysis revealed a significant main effect of suppression condition, $\beta=.24, p=.03$, indicating that participants in the suppression condition completed more of the Black-stereotypic word stems $(M=2.29$, $s=1.44)$ than did those in the control condition $(M=1.76$, $s=0.90$ ), consistent with a rebound effect. There was also a significant main effect of EMS score, $\beta=.16, p=.05$, suggesting that participants who were higher in external motivation had higher completion rates for Black-stereotypic word stems than did those low in external motivation.

Both of these main effects were, however, moderated by a marginally significant three-way interaction involving

\footnotetext{
${ }^{4}$ Stem completion rates for White-stereotypic words were unaffected by any of the predictor variables (all $p$ 's $>.20$ ) and were uncorrelated with rates for Black-stereotypic words $(r=.02, \mathrm{~ns})$.
} 

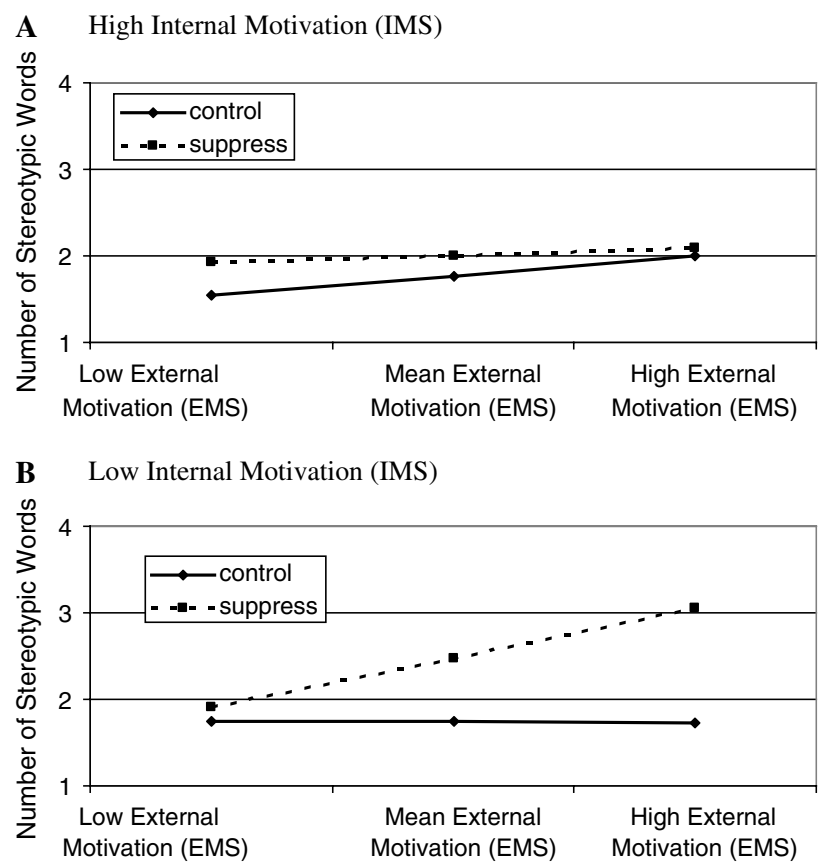

Fig. 4. Experiment 2, expression phase: mean number of Black-stereotypic word completions as a function of suppression condition and participants' scores on the external motivation scale (EMS) for participants with high scores (+1 standard deviation) on the internal motivation scale (IMS; A) and low scores ( -1 standard deviation) on the IMS (B). Regression lines estimated at points $-1,0$, and +1 standard deviation from the mean EMS scores.

suppression condition, EMS scores, and IMS scores, $\beta=-.08, p=.07$ (see Fig. 4). Although the three-way interaction did not reach standard levels of significance, the hypotheses were specific with regard to expected the pattern of results. Thus, subsequent analyses were conducted on the simple slopes (computed at \pm 1 standard deviation from the mean EMS and IMS scores). These analyses suggested that, among participants low in external motivation, suppression condition had no significant effect regardless of IMS scores ( $\beta \mathrm{s}=.08$ and .19 , ns, for low and high IMS participants, respectively). In contrast, the effect of suppression on higher EMS participants depended on their scores on the IMS. Specifically, while participants with high IMS scores were apparently unaffected by suppression instructions, $\beta=.04$, ns, those with low IMS scores showed a significant increase in stereotype use following suppression, $\beta=.65, p<.01$. No other effects were significant. Thus, consistent with hypotheses, participants who were highly motivated by external factors to respond with out prejudice displayed a significant rebound effect, but only if they did not have corresponding internal motives to respond in a non-prejudiced manner.

\section{Discussion}

In a conceptual replication of Experiment 1, participants in this experiment varied in their compliance with instructions to suppress racial stereotypes. Those who were high in external motivation generated less stereotypic descriptions in the suppression phase when asked to avoid stereotyping. In contrast, participants low in external motivation failed to reduce their level of stereotyping when asked to suppress. Consequently, only participants who were high in external motivation to respond without prejudice experienced a post-suppression rebound effect, as evidenced by their higher rates of Black-stereotypic word stem completions. Importantly, this effect was only observed for participants who were low in internal motivation to respond without prejudice, replicating past findings (Gordijn et al., 2004).

\section{General discussion}

Stereotype suppression has been the focus of a significant amount of research, presumably because of its likely use among people who wish to avoid the influence of stereotypes. Yet these experiments call into question the prevalence of suppression as a strategy used by most people. Indeed, when confronted with explicit requests to engage in suppression, only participants who were highly motivated to control prejudice (Experiment 1) or high in external motivation to respond without prejudice (Experiment 2) decreased their use of stereotypes. When suppression does occur, the likelihood of subsequent stereotype rebound is also constrained by motivational factors. Among those participants who complied with suppression instructions, only those who were highly prejudiced (Experiment 1) or low in internal motivation to respond without prejudice (Experiment 2) showed evidence of increased stereotype accessibility. Thus, the prevalence of stereotype rebound effects may be less than previously believed.

In both of the experiments reported here, participants who were relatively unprejudiced and/or highly internally motivated to respond without prejudice, but who lacked (external) motivation to avoid behaving in a prejudiced way, continued to use stereotypes even when explicitly instructed to avoid using them. This is somewhat surprising in light of the common assumption that unprejudiced (and internally motivated) individuals should be more likely to suppress social stereotypes than their prejudiced counterparts (Monteith, Sherman, \& Devine, 1998a). There are at least two possible explanations for these participants' continued production of stereotypic responses during the suppression phase. First, they may be generally less aware that their beliefs about racial outgroups are stereotypic. Thus, when they are instructed to avoid using stereotypes, they do not actively suppress their beliefs, as these beliefs are deemed to be irrelevant to the stereotype. Although this possibility cannot be ruled out on the basis of the present research, it seems somewhat unlikely in light of prior research suggesting widely shared knowledge of racial stereotypes (e.g., Devine, 1989).

Alternatively, individuals with unprejudiced beliefs combined with a lack of motivation to control prejudice (and those with high internal motivation but low external motivation) may be unlikely to apply stereotypes even in the absence of situational cues to avoid stereotyping. Either 
because stereotypes are not automatically activated for such individuals (e.g., Kawakami, Dion, \& Dovidio, 1998) or because they are habitually suppressed (e.g., Moskowitz et al., 1999), a floor effect in stereotypic responses may have been observed for participants in these categories. Although stereotypicality ratings of low prejudice/low motivation and high prejudice/low motivation participants in Experiment 1 did not statistically differ, the pattern of results is consistent with this interpretation (see also Monteith et al., 1998b).

\section{Conclusions}

People often find themselves confronted with demands to actively control their use of stereotypes. These demands may stem from societal norms that require unbiased judgments and behavior or from personal standards that advocate avoidance of stereotypic influences. Recent research has focused on stereotype suppression as one of a number of strategies to control stereotype use. The experiments reported here highlight the fact that the source of one's motivation to avoid stereotyping largely determines both the extent to which stereotypes will be effectively suppressed, as well as the longer-term consequences of suppression.

\section{References}

Bargh, J. A., \& Pietromonaco, P. (1982). Automatic information processing and social perception: the influence of trait information presented outside of conscious awareness on impression formation. Journal of Personality and Social Psychology, 43, 437-449.

Biernat, M., \& Manis, M. (1994). Shifting standards and stereotype-based judgments. Journal of Personality and Social Psychology, 66, 5-20.

Devine, P. G. (1989). Stereotypes and prejudice: their automatic and controlled components. Journal of Personality and Social Psychology, 56, $5-18$.

Devine, P. G., Plant, E. A., Amodio, D. M., Harmon-Jones, E., \& Vance, S. L. (2002). The regulation of explicit and implicit race bias: the role of motivations to respond without prejudice. Journal of Personality and Social Psychology, 82, 835-848.

Dunton, B. C., \& Fazio, R. H. (1997). An individual difference measure of motivation to control prejudiced reactions. Personality and Social Psychology Bulletin, 23, 316-326.

Fazio, R. H., \& Hilden, L. E. (2001). Emotional reactions to a seemingly prejudiced response: the role of automatically activated racial attitudes and motivation to control prejudiced reactions. Personality and Social Psychology Bulletin, 27, 538-549.

Fazio, R. H., Jackson, J. R., Dunton, B. C., \& Williams, C. J. (1995). Variability in automatic activation as an unobtrusive measure of racial atti- tudes: a bona fide pipeline? Journal of Personality and Social Psychology, 69, 1013-1027.

Galinksy, A. D., \& Moskowitz, G. B. (2000). Perspective-taking: decreasing stereotype expression, stereotype accessibility, and in-group favoritism. Journal of Personality and Social Psychology, 78, 708-724.

Gordijn, E. H., Hindriks, I., Koomen, W., Dijksterhuis, A., \& van Knippenberg, A. (2004). Consequences of stereotype suppression and internal suppression motivation: a self-regulation approach. Personality and Social Psychology Bulletin, 30, 212-224.

Hodson, G., \& Dovidio, J. F. (2001). Racial prejudice as a moderator of stereotype rebound: a conceptual replication. Representative Research in Social Psychology, 25, 1-8.

Kawakami, K., Dion, K. L., \& Dovidio, J. F. (1998). Racial prejudice and stereotype activation. Personality and Social Psychology Bulletin, 24, 407-416.

Macrae, C. N., Bodenhausen, G. V., \& Milne, A. B. (1998). Saying no to unwanted thoughts: self-focus and the regulation of mental life. Journal of Personality and Social Psychology, 74, 578-589.

Macrae, C. N., Bodenhausen, G. V., Milne, A. B., \& Jetten, J. (1994). Out of mind but back in sight: stereotypes on the rebound. Journal of Personality and Social Psychology, 67, 808-817.

McConahay, J. B. (1986). Modern racism, ambivalence, and the modern racism scale. In S. L. Gaertner \& J. F. Dovidio (Eds.), Prejudice, discrimination, and racism (pp. 91-125). New York: Harcourt Brace.

Monteith, M. J., Sherman, J. W., \& Devine, P. G. (1998a). Suppression as a stereotype control strategy. Personality and Social Psychology Review, 2, 63-82.

Monteith, M. J., Spicer, C. V., \& Tooman, G. D. (1998b). Consequences of stereotype suppression: stereotypes on AND not on the rebound. Journal of Experimental Social Psychology, 34, 355-377.

Moskowitz, G. B., Gollwitzer, P. M., Wasel, W., \& Schaal, B. (1999). Preconscious control of stereotype activation through chronic egalitarian goals. Journal of Personality and Social Psychology, 77, 167-184.

Plant, E. A. (2004). Responses to interracial interactions over time. Personality and Social Psychology Bulletin, 30, 1458-1471.

Plant, E. A., \& Devine, P. G. (1998). Internal and external motivation to respond without prejudice. Journal of Personality and Social Psychology, 75, 811-832.

Plant, E. A., \& Devine, P. G. (2001). Responses to other-imposed proBlack pressure: acceptance or backlash? Journal of Experimental Social Psychology, 37, 486-501.

Srull, T. K., \& Wyer, R. S., Jr. (1979). The role of category accessibility in the interpretation of information about persons: some determinants and implications. Journal of Personality and Social Psychology, 37, $1660-1672$.

Towles-Schwen, T., \& Fazio, R. H. (2003). Choosing social situations: the relations between automatically activated racial attitudes and anticipated comfort interacting with African Americans. Personality and Social Psychology Bulletin, 29, 170-182.

Wittenbrink, B., Judd, C. M., \& Park, B. (1997). Evidence for racial prejudice at the implicit level and its relationship with questionnaire measures. Journal of Personality and Social Psychology, 72, 262-274.

Wyer, N. A., Sherman, J. W., \& Stroessner, S. J. (1998). The spontaneous suppression of racial stereotypes. Social Cognition, 16, 340-352.

Wyer, N. A., Sherman, J. W., \& Stroessner, S. J. (2000). The roles of motivation and ability in controlling the consequences of stereotype suppression. Personality and Social Psychology Bulletin, 26, 13-25. 